ApJ Letters, Vol. 745, January 2012, in Press

Preprint typeset using $\mathrm{L}_{\mathrm{T}} \mathrm{EX}$ style emulateapj v. 5/2/11

\title{
FIRST SDO/AIA OBSERVATION OF SOLAR PROMINENCE FORMATION FOLLOWING AN ERUPTION: MAGNETIC DIPS AND SUSTAINED CONDENSATION AND DRAINAGE
}

\author{
Wei Liu ${ }^{12}$, Thomas E. Berger ${ }^{1}$, And B. C. Low ${ }^{3}$ \\ ApJ Letters, Vol. 745, January 2012, in press
}

\begin{abstract}
Imaging solar coronal condensation forming prominences was difficult in the past, a situation recently changed by Hinode and $S D O$. We present the first example observed with SDO/AIA, in which material gradually cools through multiple EUV channels in a transequatorial loop system that confines an earlier eruption. Nine hours later, this leads to eventual condensation at the dips of these loops, forming a moderate-size prominence of $\sim 10^{14} \mathrm{~g}$, to be compared to the characteristic $10^{15} \mathrm{~g}$ mass of a CME. The prominence mass is not static but maintained by condensation at a high estimated rate of $10^{10} \mathrm{~g} \mathrm{~s}^{-1}$ against a comparable, sustained drainage through numerous vertical downflow threads, such that $96 \%$ of the total condensation $\left(\sim 10^{15} \mathrm{~g}\right)$ is drained in approximately one day. The mass condensation and drainage rates temporally correlate with the total prominence mass. The downflow velocity has a narrow Gaussian distribution with a mean of $30 \mathrm{~km} \mathrm{~s}^{-1}$, while the downward acceleration distribution has an exponential drop with a mean of $\sim 1 / 6 g_{\odot}$, indicating a significant canceling of gravity, possibly by the Lorentz force. Our observations show that a macroscopic quiescent prominence is microscopically dynamic, involving the passage of a significant mass through it, maintained by a continual mass supply against a comparable mass drainage, which bears important implications for CME initiation mechanisms in which mass unloading is important.

Subject headings: Sun: activity —Sun: corona — Sun: filaments, prominences
\end{abstract}

\section{INTRODUCTION}

Solar prominences are over dense and cool material in the tenuous, hot corona (Tandberg-Hanssen 1995; Martin 1998; Labrosse et al. 2010; Mackay et al. 2010). Proposed mechanisms for prominence formation include: (1) chromospheric transport by siphon flows (Pikel'Ner 1971; Engvold \& Jensen 1977), magnetic reconnection (Litvinenko \& Martin 1999; Wang 1999), or magnetic flux emergence ( $\mathrm{Hu} \& \mathrm{Liu}$ 2000; Okamoto et al. 2009), and (2) coronal condensation due to a diversity of radiatively-driven thermal instabilities (Parker 1953; Priest 1982, p.277) occurring in current sheets (Smith \& Priest 1977; Titov et al. 1993) and coronal loops (Wu et al. 1990; Mok et al. 1990; Antiochos \& Klimchuk 1991; Choe \& Lee 1992; Karpen et al. 2003, 2005; Luna Bennasar et al.|2012).

Prominence formation by condensation was once questioned because a quiet, static corona is an inadequate mass source (Saito \& Tandberg-Hanssen 1973). This does not apply to the solar corona that is known to be dynamic with mass being continually replenished, say, by footpoint heating driving chromospheric evaporation (Karpen \& Antiochos 2008; Xia et al. 2011), by ubiquitous spicules (De Pontieu et al.|2011), or by thermal convection involving emerging magnetic bubbles (Berger et al. 2011).

Condensation was seen in the so-called "cloud prominences" (Engvold 1976; Tandberg-Hanssen 1995; Lin et al. 2006; Stenborg et al. 2008) or "coronal spi-

\footnotetext{
${ }^{1}$ Lockheed Martin Solar and Astrophysics Laboratory, Building 252, 3251 Hanover Street, Palo Alto, CA 94304

2 W. W. Hansen Experimental Physics Laboratory, Stanford University, Stanford, CA 94305

${ }^{3}$ High Altitude Observatory, National Center for Atmospheric Research, P.O. Box 3000, Boulder, CO 80307
}

ders" (Allen et al. 1998) with material streaming out along curved trajectories. It was also observed in cooling loops (Landi et al. 2009) and proposed to produce coronal rain (Schrijver 2001; Müller et al. 2003). However, quantitative analysis of prominence condensation has been hampered by the high off-limb scattered light of ground-based instruments and limited spatial/temporal resolution or field of view (FOV) of previous space instruments.

This situation has changed with the launches of the Hinode and Solar Dynamics Observatory (SDO). SDO's Atmospheric Imaging Assembly (AIA; Lemen et al. 2011) offers unprecedented capabilities with a full Sun FOV at 12 s cadence and 1". 5 resolution. Here we present a first AIA observation of prominence formation, involving magnetic dips and plasma condensing there to build up the prominence mass and balance sustained drainage. Observations of this kind may provide answers to three long-standing questions:

1. How do condensation and drainage control the mass budget of a prominence?

2. What are the favorable plasma and magnetic field conditions at the micro and macroscopic scales of a prominence?

3. What is the relationship between prominences and other solar activities, such as CMEs and flares?

\section{OBSERVATIONAL OVERVIEW: PROMINENCE FORMATION AFTER CONFINED ERUPTION}

On 2010/11/25 at about 21:10 UT, a confined eruption is observed by $S D O /$ AIA and STEREO A in NOAA active region (AR) 11126 (Figures 1(a)-(c); Animations $1(\mathrm{~A})$ and $1(\mathrm{~B}))$. Most of the pre-existing promi- 

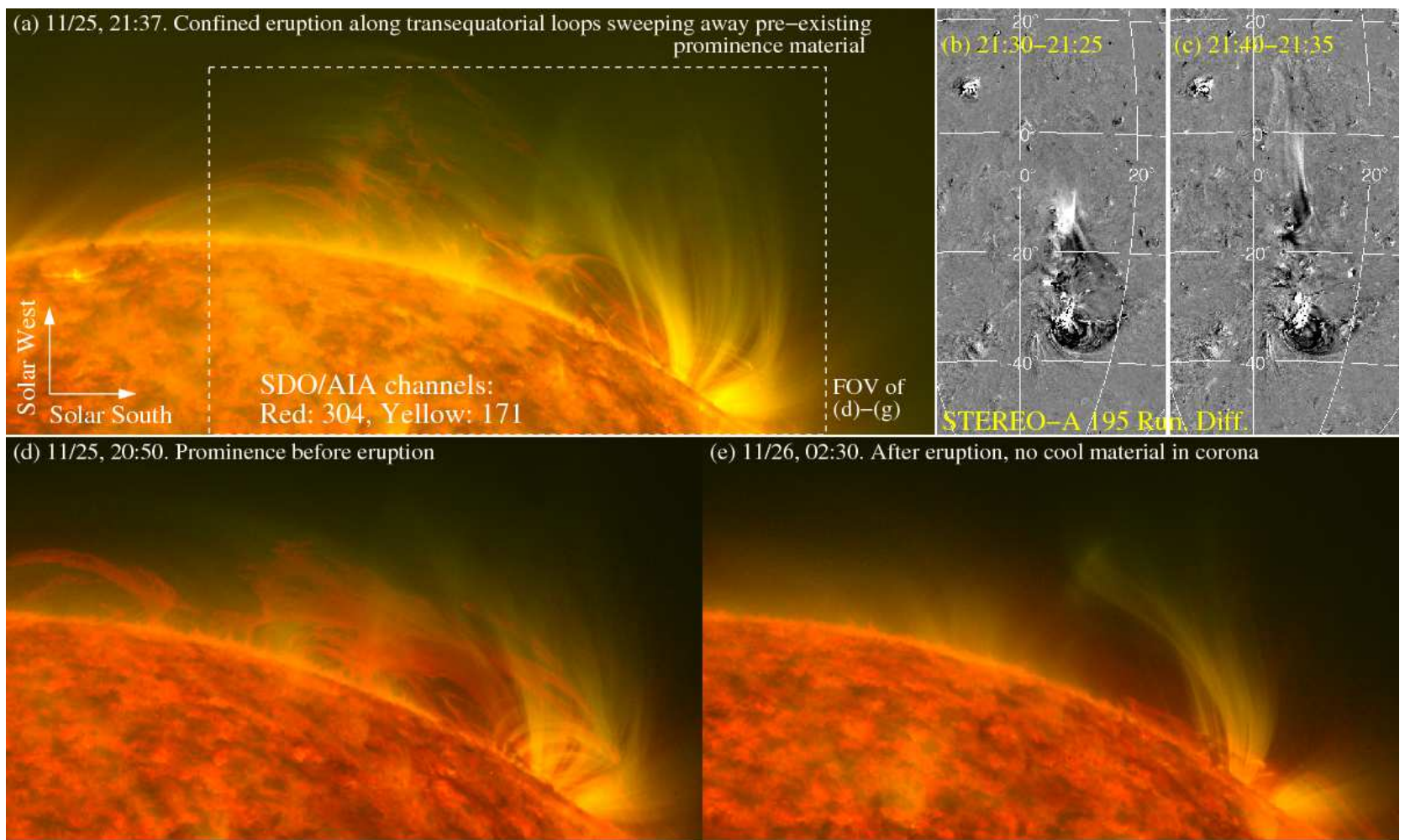

(e) 11/26, 02:30. After eruption, no cool material in corona

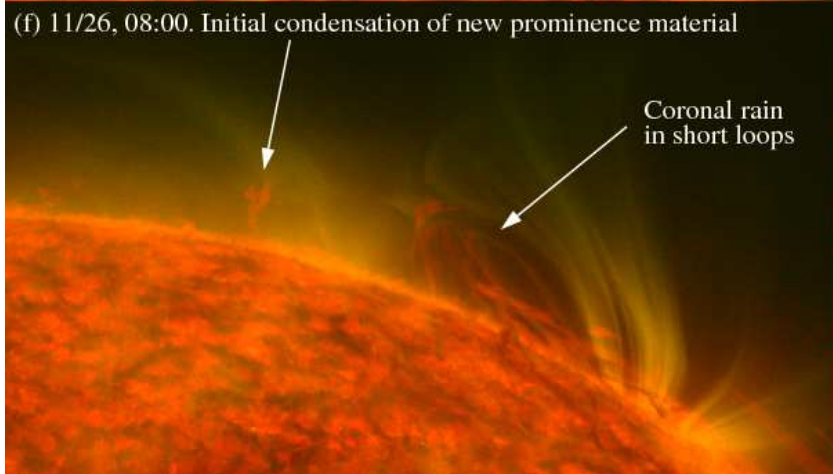

(g) $11 / 26,14: 20$. New prominence formed

FIG. 1. - (a) Composite AIA 304 (red) and $171 \AA$ (yellow) image, rotated to Solar West up, showing a confined eruption (Animation 1(A)). (b) and (c) $195 \AA$ running difference images from STEREO A, $85^{\circ}$ ahead of the Earth, observing the eruption from a top view (Animation 1(B)). Note the bulging coronal volume and the ejected material forming a rubber syringe shape. (d)-(g) Same as (a) but for a smaller FOV (boxed region) showing the event sequence (Animations 1(C) and 1(D)).

nence material is swept away along transequatorial loops at $\gtrsim 130 \mathrm{~km} \mathrm{~s}^{-1}$ and some lands on the other hemisphere.

At 2010/11/26 05:00 UT, the first sign of new, cool $304 \AA$ material is observed at the dips in the active region loops and consequently slides downward along underlying loops as coronal rain (Figure1(f); Animations 1(C)). Two hours later, a nearby condensation at greater rates leads to the formation of a new prominence (Figures 1(f) and $(\mathrm{g}))$. After another day, the prominence gradually shrinks and eventually disappears behind the limb.

In this Letter, we focus on the condensation and mass drainage in the new prominence, leaving other interesting aspects of this event for future investigation.

\section{INITIAL CONDENSATION AT MAGNETIC DIPS IN TRANSEQUATORIAL LOOPS}

Let us first examine the magnetic environment. Prior to the eruption, there are transequatorial loops of various lengths, best seen at $171 \AA$ in Figure 2(a) and Animation
1(A). The limb location of the event does not allow accurate determination of the magnetic configuration, but potential field extrapolations up to a week earlier indicate the persistence of such loops that connect the positive trailing polarity of AR 11126 in the south and the diffuse negative polarity in the north 5

After the eruption, these loops fade and gradually reappear. Since 11/26 05:00 UT, one can can identify an apparent depression, likely formed in projection by shallow dips of numerous loops (Figure 2, left), where the initial condensation occurs shortly at 07:00 UT. The cool $304 \AA$ material forms a "V"-shape (Figure 2, right), whose lower tip progressively drops and eventually drains down in a nearly vertical direction, suggesting magnetic reconnection (as sketched here) or cross-field slippage (Low et al. 2012b, in preparation).

\footnotetext{
${ }^{4}$ http://sohowww.nascom.nasa.gov/sdb/packages/pfss/l1q_synop

5 see magnetograms on 11/18 at http://www.solarmonitor.org
} 

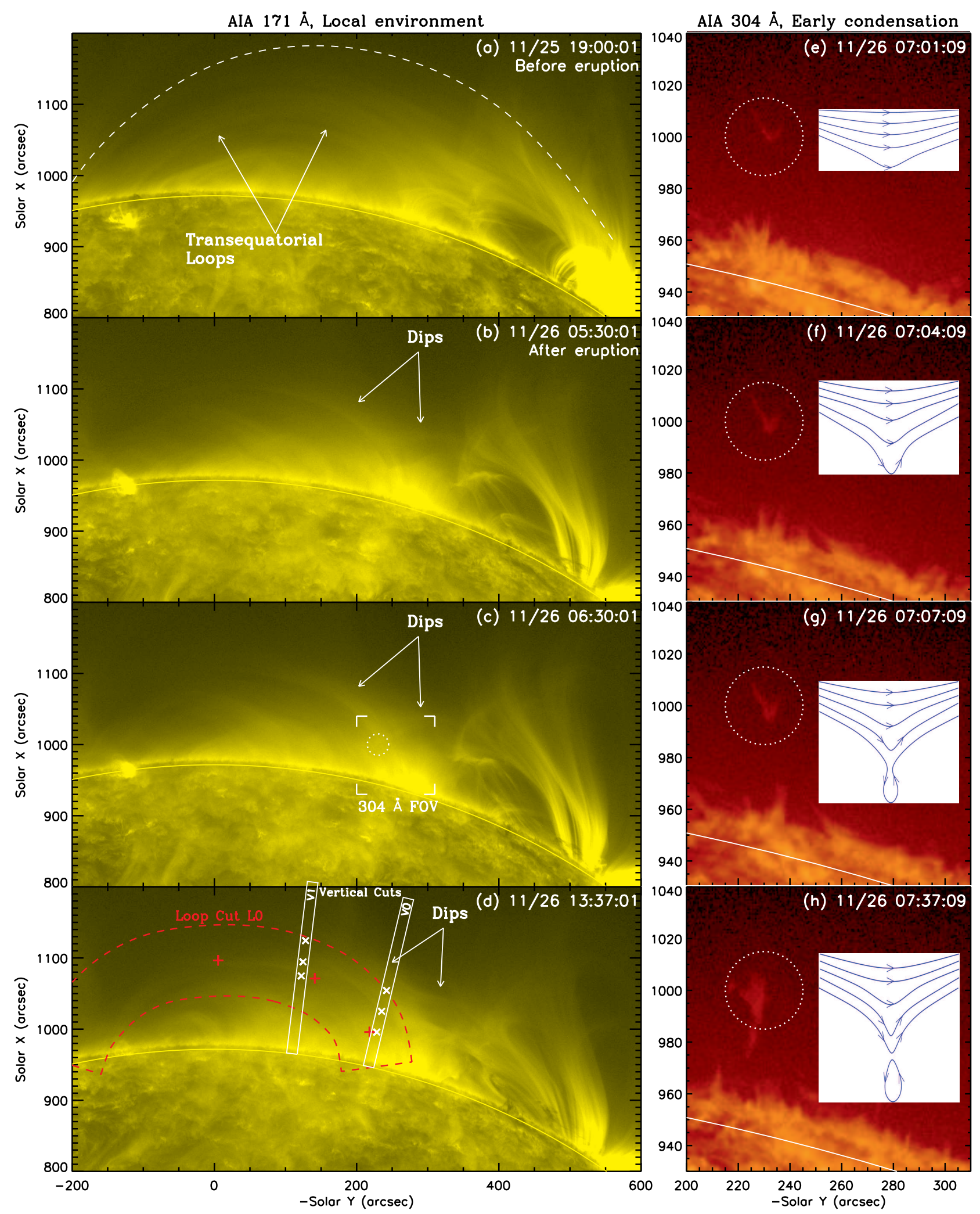

FIG. 2.- Left: AIA $171 \AA$ images showing dips among elongated transequatorial loops. Superimposed in (d) are cuts used to obtain space-time diagrams in Figure 3 Right: Enlarged AIA $304 \AA$ images showing the "V"-shaped initial condensation at the lowest dip. The insets sketch a candidate magnetic configuration. 


\section{POST-ERUPTION COOLING IN TRANSEQUATORIAL LOOPS}

To track the cooling process and infer the origin of the prominence mass, we placed a $100^{\prime \prime}\left(1^{\prime \prime}=716 \mathrm{~km}\right)$ wide cut L0 along the transequatorial loops to the north of the dips and two vertical cuts of $15^{\prime \prime}$ wide and $243^{\prime \prime}$ tall from the limb across these loops at the lowest dip (V0) and further north (V1; Figure 2(d); cuts to the south of the dips yielded similar results). By averaging image pixels across each cut, we obtained space-time diagrams as shown in Figure 3 together with selected temporal profiles.

We find a general trend of successive brightening after the eruption at 193,171 , and then $304 \AA$, each delayed by $2-5$ hours. This indicates gradual cooling across the characteristic temperatures at the response peaks of these broadband channels, from $1.6 \times 10^{6}$ to $7.9 \times 10^{5}$ and $7.9 \times 10^{4} \mathrm{~K}$ (Boerner et al. 2011). At $193 \AA$, the initial brightening likely results from mass being replenished after the eruption and/or cooling from even higher temperatures; the later darkening at 9:00 UT and heights $30^{\prime \prime}-80^{\prime \prime}$ is primarily due to absorption by the cool prominence material.

Condensation of the $304 \AA$ material occurs only at low altitudes $\left(<100^{\prime \prime}\right)$ near the dips, while cooling from higher temperatures takes place almost throughout the entire transequatorial loop system involving a vast volume (Figure 3, left). This implies that these loops likely feed mass to the prominence forming at their dips that, partly because of the stretched and possibly sheared magnetic field lines (say, near a polarity inversion line) and thus reduced field-aligned thermal conduction, may facilitate further cooling and condensation (Low et al. 2012a, in preparation). We detected no systematic cooling flow in the loops though, possibly due to the insensitivity of AIA (passband imager) to subtle flows without distinct mass blobs, except for occasional brief downflows and upflows of $304 \AA$ material (Figures 3 (g)). The $9 \mathrm{hr}$ elapsed from the eruption to the initial condensation is on the same order of magnitude as the condensation timescales in thermal nonequilibrium simulations (Karpen et al. 2005).

Condensation at $304 \AA$ occurs first at a height of $60^{\prime \prime}$ and progresses toward higher loops at $2 \mathrm{kms}^{-1}$ $\left(\gg 0.3 \mathrm{kms}^{-1}\right.$, the projected speed of the solar rotation there), with a similar trend at $171 \AA$ (Figures $3(\mathrm{e})$ and $(\mathrm{h})$ ). We speculate that those low-lying loops condense first because of their shorter lengths Karpen \& Antiochos 2008) and/or their greater densities from gravitational stratification (as radiative loss $\left.\propto n^{2}\right)$.

\section{MASS BUDGET OF CONDENSATION AND DRAINAGE}

In this prominence, we find no sign of rising bubbles (Berger et al. 2008, 2010, 2011) that can contribute to the prominence mass. If we further neglect other possible mass sources, the interplay between the mass condensation rate $\dot{M}_{\text {cond }}$ and downflow rate $\dot{M}_{\text {drain }}$ would determine the prominence mass change rate

$$
\dot{M}_{\text {prom }}=\dot{M}_{\text {cond }}-\dot{M}_{\text {drain }}
$$

The condensation rate $\dot{M}_{\text {cond }}$, which cannot be measured directly, can thus be inferred by measuring $\dot{M}_{\text {prom }}$ and $\dot{M}_{\text {drain }}$ independently, as described below in three steps.

\subsection{Prominence Mass Budget $\dot{M}_{\text {prom }}$}

This prominence consists of numerous nearly vertical threads of typically $d_{\text {thrd }}=2^{\prime \prime}(1.43 \mathrm{Mm})$ thick (see also Berger et al. 2008). The observed optically thick $304 \AA$ emission originates from a layer known as the prominence-coronal transition region (PCTR) surrounding a denser, cooler $\mathrm{H} \alpha$ emitting core (e.g., Lin et al. 2005; Lin 2011), later seen in Mauna Loa data (not shown). However, lacking sufficient $\mathrm{H} \alpha$ coverage of the event, we chose to use a typical PCTR electron density $n_{\mathrm{e}}=8 \times 10^{9} \mathrm{~cm}^{-3}$ (Labrosse et al. 2010) and thus $\rho=n_{\mathrm{e}} m_{\mathrm{p}}=1.3 \times 10^{-14} \mathrm{~g} \mathrm{~cm}^{-3}$ as a lower limit for the entire prominence. We also conservatively assumed that the observed threads do not overlap each other.

We selected a sector-shaped area to enclose the bulk of the prominence (see Figure 4(c)). The sector covers a height range of $25^{\prime \prime}-110^{\prime \prime}$ above the limb and a latitude range of $8.3^{\circ}\left(144^{\prime \prime}\right.$ wide on its bottom edge). We then integrated the area $A_{\text {prom }}$ occupied by the prominence material inside the sector for all pixels above a threshold selected at $3 \sigma$ above background. We obtained a lower limit to the prominence mass

$$
M_{\text {prom }}=\rho A_{\text {prom }} d_{\text {thrd }},
$$

as shown in Figure 5(a). After the initial condensation, the prominence mass continues to increase and reaches the maximum at 16:01 UT,

$$
M_{\text {prom, } \max }=6.0 \times 10^{13} \mathrm{~g}
$$

which is on the same order of magnitude as that determined from coronal absorption for a prominence of similar size (Gilbert et al. 2005). Taking time derivative gives the mass change rate $\dot{M}_{\text {prom }}$ shown in Figure 5)(c), which has a mean of $\left\langle\dot{M}_{\text {prom }}\right\rangle=-5.6 \times 10^{7} \mathrm{~g} \mathrm{~s}^{-1}$ and a maximum at 11:37 UT,

$$
\dot{M}_{\text {prom }, \max }=7.6 \times 10^{9} \mathrm{~g} \mathrm{~s}^{-1} \text {. }
$$

\subsection{Mass drainage Rate $\dot{M}_{\text {drain }}$}

To estimate the drainage rate of mass that leaves the sector-shaped area, we need to obtain the downflow velocity $v(s, t)$ at position $s$ on the bottom edge of the sector and at time $t$, and then spatially integrate the mass flux crossing this edge. Here we assume that the warm PCTR and cool core of the prominence thread have the same downflow velocity (Labrosse et al. 2010). To measure $v(s, t)$, we divided the sector into 24 vertical cuts of $6^{\prime \prime}$ wide that start at $15^{\prime \prime}$ above the limb to avoid spicules and discretize the bottom sector edge into distance bins $s_{i}(i=0,1, \ldots, 23)$, as shown in Figure 4(c). For each cut, we obtained a space-time diagram for a $19 \mathrm{hr}$ duration from 11/26 07:00 to 11/27 02:00 UT.

As an example, the space-time diagram (Figures 4 (d) and (e)) of cut \#12 located near the middle of the promi-

\footnotetext{
6 The $6^{\prime \prime}$ width of the vertical cuts was chosen to be as small as possible and yet wide enough to enclose most of the prominence threads that are close to but not exactly vertical.
} 

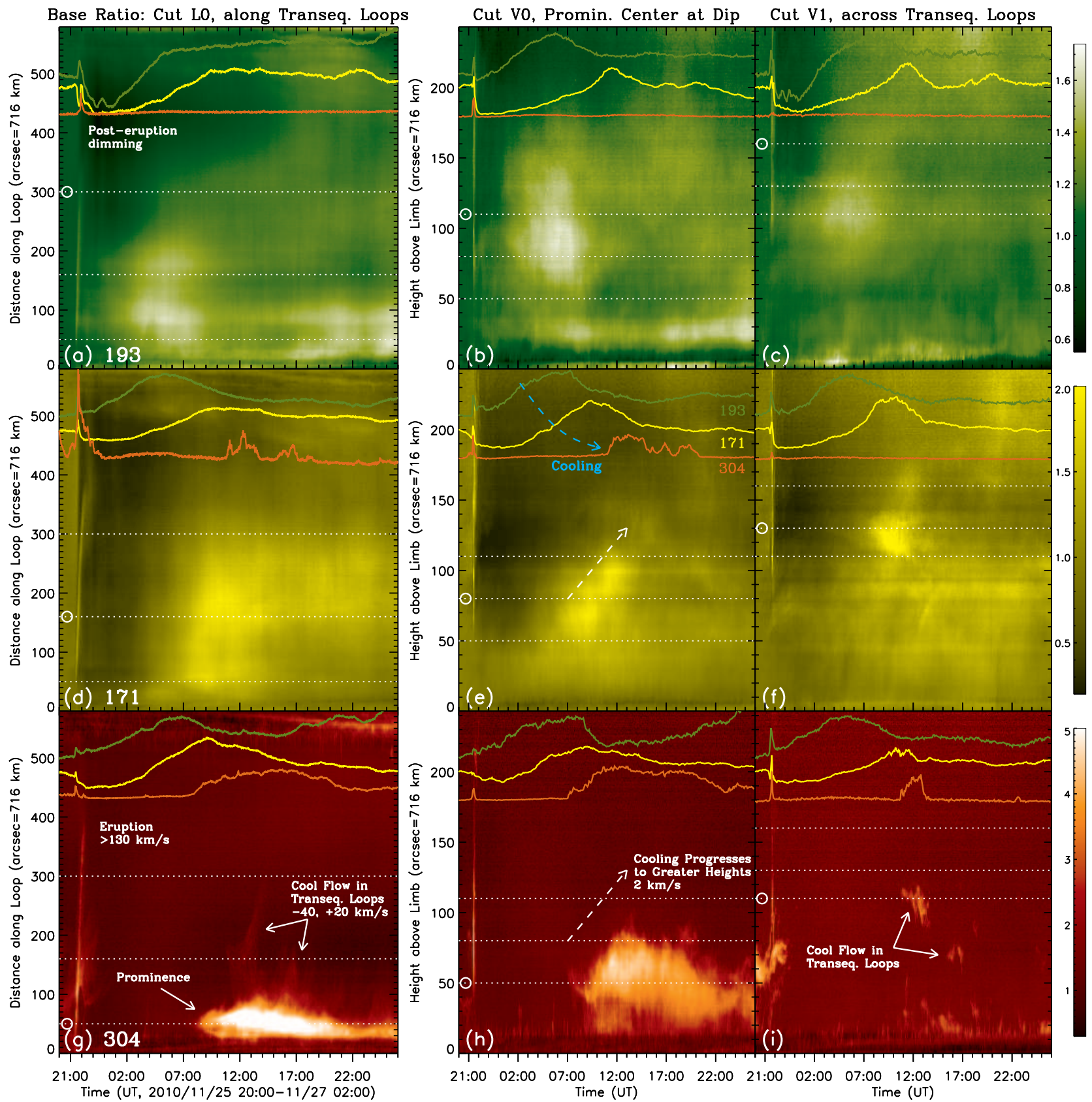

Fig. 3.- Base ratio space-time diagrams at 193 (green), 171 (yellow), and $304 \AA$ (red) from the loop shaped cut L0 (left) and vertical cuts V0 (middle) and V1 (right) shown in Figure 2 d). Three positions are selected for each cut, as marked with the plus or cross signs there, to obtain horizontal slices from the diagram here as indicated by the white-dotted lines. The resulting temporal profiles (arbitrarily scaled) in the three channels are shown in each panel for the slice marked with an open circle.

nence shows numerous, episodic downward curved trajectories. Each trajectory tracks the height of a blob of mass with time as it descends within the vertical cut. The descent covers a height range of several tens of arcseconds and lasts for a few minutes to half an hour. We fitted each well-defined trajectory with a parabolic function, as shown in Figure 4(d), and determined the average acceleration $a_{\text {prom }}$ and velocity $v_{\text {prom }}$ at the reference height $h_{\text {ref }}=25^{\prime \prime}$ (horizontal dashed line) of the bottom edge of the sector. We exhaustively repeated such fits throughout the space-time diagram, only skipping an adjacent trajectory of a similar slope within any 10 minutes interval. The resulting fits are overlaid in Figure 4(e), and the fitted velocities and accelerations are shown in Figures $4(\mathrm{f})$ and (g). Finally, we linearly interpolated the fitted velocities in time and obtained $v\left(s_{12}, t\right)$ covering the full duration.

We applied this procedure to all vertical cuts across the prominence and obtained a statistically significant sample of 874 parabolic downflow trajectories. As shown in Figure 4(h), the fitted velocities have a narrow Gaussian distribution, with a mean and median of $\left\langle v_{\text {prom }}\right\rangle=$ $30 \mathrm{kms}^{-1}$, mode of $28 \mathrm{kms}^{-1}$, and standard deviation of $13 \mathrm{~km} \mathrm{~s}^{-1}$. In contrast, the accelerations (Figure 4(i)) have an exponential distribution $\propto e^{-0.023 a}$, with a mean of $\left\langle a_{\text {prom }}\right\rangle=46 \mathrm{~ms}^{-2}=0.17 g_{\odot} \approx 1 / 6 g_{\odot}$, a standard 

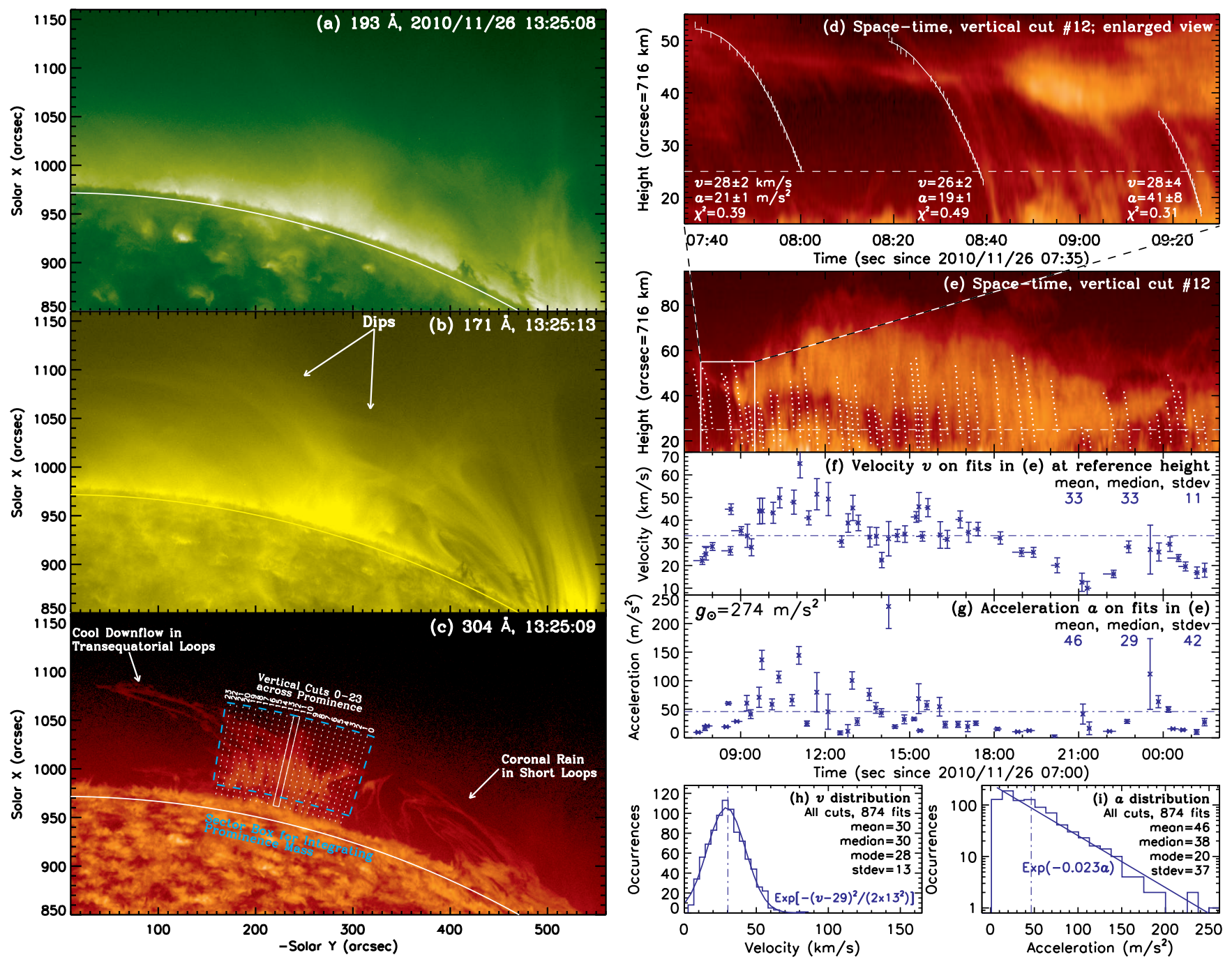

FIG. 4.- (a)-(c) Simultaneous AIA 193, 171, and $304 \AA$ images showing the neighborhood of the new prominence. (d) Enlarged spacetime diagram of (e) in the boxed region superimposed with selected fits. (e) Space-time diagram obtained from a typical vertical cut (\#12, marked with solid lines in (c); see Animation 4 for the diagrams of all vertical cuts). White dotted lines are parabolic fits to downflow trajectories. The horizontal dashed line marks the reference height $h_{\mathrm{ref}}=25^{\prime \prime}$ of the bottom edge of the sector at which downflow velocities are measured. (f) and (g) Velocities and accelerations from the fits in (d) as a function of time. Each short horizontal bar indicates the duration of the fit. (h) and (i) Histograms of velocities and accelerations from 874 measurements obtained from all 24 vertical cuts, whose distributions are fitted with a Gaussian and exponential function, respectively. The dot-dashed lines in (f)-(i) mark the corresponding mean values.

deviation of $37 \mathrm{~ms}^{-2}\left(0.14 g_{\odot}\right)$, and a small mode of $20 \mathrm{~m} \mathrm{~s}^{-2}\left(0.07 g_{\odot}\right)$, where $g_{\odot}=274 \mathrm{~m} \mathrm{~s}^{-2}$ is the solar surface gravitational constant.

After obtaining the downflow velocity $v\left(s_{i}, t\right)$ at all cuts, next we need the knowledge of the spatial width and temporal duration of each downflow stream. For the former, we assumed that within each $6^{\prime \prime}$ wide cut, no more than one vertical thread (typical width $2^{\prime \prime}$ ) undergoes downflow at any moment, giving an effective filing factor of $2^{\prime \prime} / 6^{\prime \prime}=1 / 3$. For the latter, we defined a switch function $Z\left(s_{i}, t\right)$ that describes whether downflow occurs $(Z=1)$ or not $(Z=0)$ at the $i$ th cut and time $t$, depending on whether or not the emission intensity $\left.I\left(s_{i}, t\right)\right|_{h=h_{\mathrm{ref}}}$ (e.g., Figure 4(d)) in the corresponding space-time diagram at height $h_{\text {ref }}$ is $5 \sigma$ above the pre-event background.

Finally, summing over all vertical cuts (as spatial inte- gration) yields the mass drainage rate:

$$
\dot{M}_{\text {drain }}(t)=\sum_{i=0}^{23} \rho A_{\text {thrd }} v\left(s_{i}, t\right) Z\left(s_{i}, t\right),
$$

where $A_{\text {thrd }}=d_{\text {thrd }}^{2}$ is the cross-sectional area of the thread. As shown in Figure 5(c), it has a mean

$$
\left\langle\dot{M}_{\text {drain }}\right\rangle=1.1 \times 10^{10} \mathrm{~g} \mathrm{~s}^{-1},
$$

and a maximum

$$
\dot{M}_{\text {drain, } \max }=(2.1 \pm 0.5) \times 10^{10} \mathrm{~g} \mathrm{~s}^{-1}
$$

at 11/26 14:08 UT. Integrating $\dot{M}_{\text {drain }}$ over time yields the accumulative mass drained to date $M_{\text {drain }}(t)$, as shown in Figure 5(b). At the end of the 19 hr duration, it reaches

$$
M_{\text {drain, } \max }=7.7 \times 10^{14} \mathrm{~g},
$$




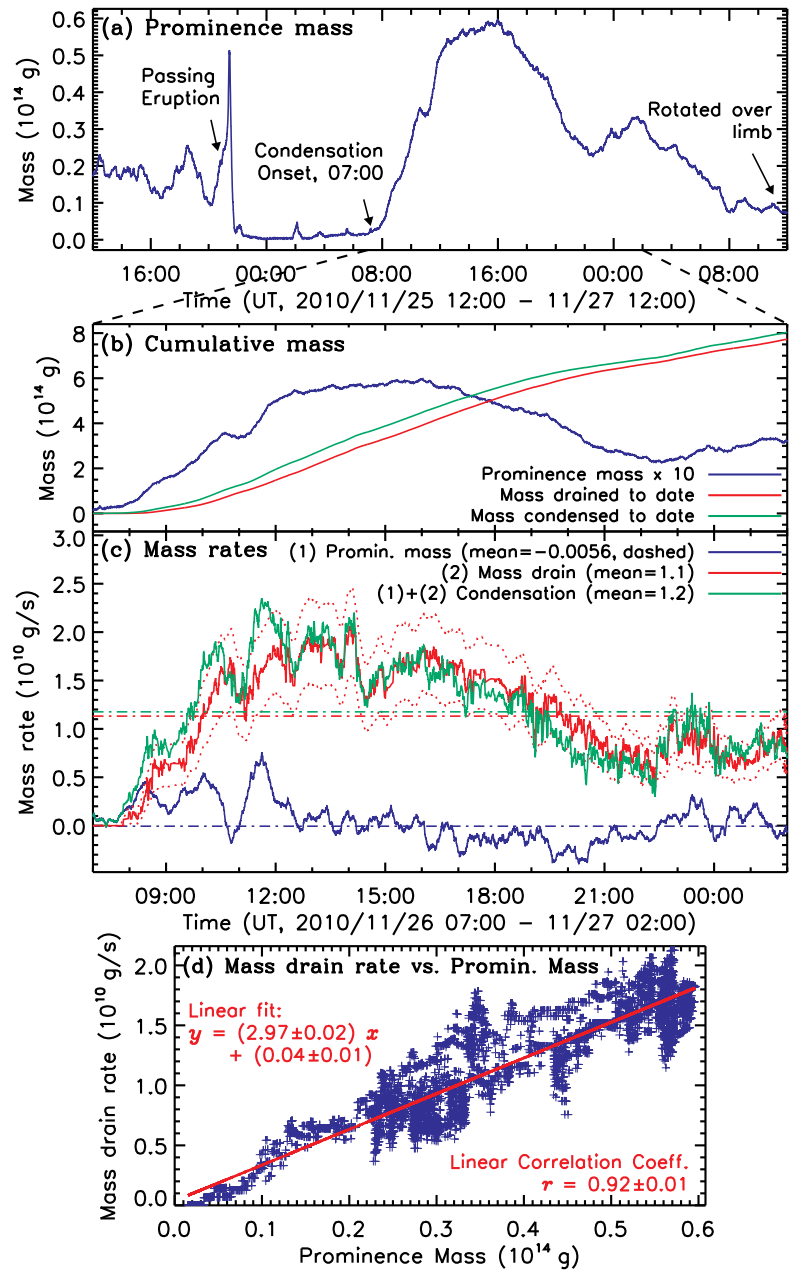

FIG. 5.- (a) Temporal variation over 2 days of the estimated prominence mass $M_{\text {prom }}$. (b) Prominence mass budget (blue, repeated from (a) but scaled by a factor of 10) and cumulative mass drained (red) and condensed (green) to date. (c) Time derivatives of the curves in (b). The red dotted lines mark the bounds of uncertainties of the mass drainage rate estimated from downflow velocity uncertainties (see, e.g., Figure 4 (f)). The horizontal dotdashed lines indicate the corresponding mean values. (d) Mass drainage rate versus prominence mass, displaying a positive linear correlation.

which is 13 times the maximum prominence mass $M_{\text {prom, } \max }$.

\subsection{Mass Condensation Rate $\dot{M}_{\text {cond }}$}

The mass conservation equation (1) then gives the condensation rate

$$
\dot{M}_{\text {cond }}=\dot{M}_{\text {prom }}+\dot{M}_{\text {drain }}
$$

as shown in Figure 5(c). It closely follows the mass drainage rate $\dot{M}_{\text {drain }}$, which dominates over the prominence mass change $\dot{M}_{\text {prom }}$ by about an order of magnitude. The condensation rate has a similar mean

$$
\left\langle\dot{M}_{\text {cond }}\right\rangle=1.2 \times 10^{10} \mathrm{~g} \mathrm{~s}^{-1},
$$

and maximum

$$
\dot{M}_{\text {cond, } \max }=(2.4 \pm 0.4) \times 10^{10} \mathrm{~g} \mathrm{~s}^{-1}
$$

at 11/26 11:37 UT. The cumulative mass condensed in $19 \mathrm{hr}$ is

$$
M_{\text {cond, } \max }=8.0 \times 10^{14} \mathrm{~g},
$$

about the same as the mass drained to date.

We find two episodes of rapid condensation and mass drainage, starting at 11/26 07:00 and 22:00 UT, which coincide with the variations of the prominence mass (Figure 5(b)). In fact, the drainage rate is positively correlated with the total prominence mass, with a linear correlation coefficient of $r=0.92 \pm 0.1$ (Figure [5(d)).

\section{SUMMARY AND DISCUSSION}

We have presented new evidence of coronal condensation that leads to prominence formation at magnetic dips after a confined eruption. Our major findings and their implications are as follows.

1. The estimated average mass condensation rate in the prominence is $\approx 10^{10} \mathrm{~g} \mathrm{~s}^{-1}$, giving an accumulative mass of $\sim 10^{15} \mathrm{~g}$ in $19 \mathrm{hr}$. Most (96\%) of this mass, an order of magnitude more than the instantaneous total mass of the prominence, is drained as vertical threads. In other words, a macroscopically quiescent prominence is a microscopically dynamic object, with a total mass determined by the delicate balance between a significant mass supply and a drainage by downflows. This was suggested long ago (e.g., Priest \& Smith 1979; Tandberg-Hanssen 1995), but to the best of our knowledge, our observations provide the first quantitative evidence in terms of the prominence mass budget.

2. The mass condensation and drainage rates are very close and linearly correlated with the total prominence mass. Thus, the more material the prominence has, the more mass it is capable of draining. Theoretical considerations of energy balance by Low et al. (2012b, in preparation) indicates that the total mass is a critical parameter that determines how fast condensation takes place.

3. The prominence downflows are much slower than free fall, with an average acceleration of $\left\langle a_{\text {prom }}\right\rangle \approx 1 / 6 / g_{\odot}$, similar to that found by Chae (2010). This shows that gravity in the prominence is countered significantly, possibly by an upward Lorentz force (e.g., Low \& Petrie 2005; van Ballegooijen \& Cranmer 2010) from the horizontal component of the magnetic field, as indicated by polarimetric measurements (Casini et al. 2003 ), or by transverse wave generated pressure as originally suggested for braking coronal rain (Antolin \& Verwichte 2011).

4. After hours of cooling from $\sim 10^{6} \mathrm{~K}$ throughout almost the entire transequatorial loop system that confined the early eruption, the initial prominence condensation $\left(\sim 10^{5} \mathrm{~K}\right)$ occurs at the loop dips and consequently progresses toward greater heights. This suggests that these large-scale loops may function like a funnel with a vast collecting volume that feeds mass to the prominence, and the dips likely provide favorable conditions for further cooling and condensation (cf., Karpen et al. 2001).

5. The preceding eruption might be independent of the prominence reformation or have provided 
heated material to make the confining transequatorial loops overdense, favoring radiative cooling and possibly responsible for other post-eruption prominence formations (Lin et al. 2006; Wang et al. 2007).

6. We propose that the passage of mass on the order of $10^{15} \mathrm{~g}$ /day, comparable to a CME mass, could be a general property of prominences exhibiting active drainage. If the "magneto-thermal convection" suggested by Berger et al. (2011) in prominencecavity structures also involves the drainage of such a large mass, its weight is energetically significant for holding a magnetic flux rope in the cavity until it erupts into a CME (Low 2001; Zhang \& Low
2005).

This study and future observations of coronal condensation may provide further insights into prominences as an MHD phenomenon, with answers to the long-standing questions noted in the Introduction.

WL was supported by AIA contract NNG04EA00C. TEB was supported by the Solar-B FPP contract NNM07AA01C at Lockheed Martin. The National Center for Atmospheric Research is sponsored by the National Science Foundation. We thank the anonymous referee for critical comments and Sara Martin, Yong Lin, Jingxiu Wang, and Roberto Casini for helpful discussions.

\section{REFERENCES}

Allen, U. A., Bagenal, F., \& Hundhausen, A. J. 1998, in Astronomical Society of the Pacific Conference Series, Vol. 150, IAU Colloq. 167: New Perspectives on Solar Prominences, ed. D. F. Webb, B. Schmieder, \& D. M. Rust, 290

Antiochos, S. K. \& Klimchuk, J. A. 1991, ApJ, 378, 372

Antolin, P. \& Verwichte, E. 2011, ApJ, 736, 121

Berger, T., et al. 2011, Nature, 472, 197

Berger, T. E., et al. 2008, ApJ, 676, L89

Berger, T. E., et al. 2010, ApJ, 716, 1288

Boerner, P., et al. 2011, Sol. Phys., 316

Casini, R., López Ariste, A., Tomczyk, S., \& Lites, B. W. 2003, ApJ, 598, L67

Chae, J. 2010, ApJ, 714, 618

Choe, G. S. \& Lee, L. C. 1992, Sol. Phys., 138, 291

De Pontieu, B., McIntosh, S. W., Carlsson, M., Hansteen, V. H., Tarbell, T. D., Boerner, P., Martinez-Sykora, J., Schrijver, C. J., \& Title, A. M. 2011, Science, 331, 55

Engvold, O. 1976, Sol. Phys., 49, 283

Engvold, O. \& Jensen, E. 1977, Sol. Phys., 52, 37

Gilbert, H. R., Holzer, T. E., \& MacQueen, R. M. 2005, ApJ, 618,524

Hu, Y. Q. \& Liu, W. 2000, ApJ, 540, 1119

Karpen, J. T. \& Antiochos, S. K. 2008, ApJ, 676, 658

Karpen, J. T., Antiochos, S. K., Hohensee, M., Klimchuk, J. A., \& MacNeice, P. J. 2001, ApJ, 553, L85

Karpen, J. T., Antiochos, S. K., Klimchuk, J. A., \& MacNeice, P. J. 2003, ApJ, 593, 1187

Karpen, J. T., Tanner, S. E. M., Antiochos, S. K., \& DeVore, C. R. 2005, ApJ, 635, 1319

Labrosse, N., Heinzel, P., Vial, J.-C., Kucera, T., Parenti, S., Gunár, S., Schmieder, B., \& Kilper, G. 2010, Space Sci. Rev. 151,243

Landi, E., Miralles, M. P., Curdt, W., \& Hara, H. 2009, ApJ, 695, 221

Lemen, J. R., et al. 2011, Sol. Phys., 106

Lin, Y. 2011, Space Sci. Rev., 158, 237

Lin, Y., Engvold, O., Rouppe van der Voort, L., Wiik, J. E., \& Berger, T. E. 2005, Sol. Phys., 226, 239

Lin, Y., Martin, S. F., \& Engvold, O. 2006, in Bulletin of the American Astronomical Society, Vol. 38, AAS/Solar Physics Division Meeting \#37, 219
Litvinenko, Y. E. \& Martin, S. F. 1999, Sol. Phys., 190, 45

Low, B. C. 2001, J. Geophys. Res., 106, 25141

Low, B. C. \& Petrie, G. J. D. 2005, ApJ, 626, 551

Luna Bennasar, M., Karpen, J., \& DeVore, C. R. 2012, ApJ, in press

Mackay, D. H., Karpen, J. T., Ballester, J. L., Schmieder, B., \& Aulanier, G. 2010, Space Sci. Rev., 151, 333

Martin, S. F. 1998, Sol. Phys., 182, 107

Mok, Y., Drake, J. F., Schnack, D. D., \& van Hoven, G. 1990, ApJ, 359, 228

Müller, D. A. N., Hansteen, V. H., \& Peter, H. 2003, A\&A, 411, 605

Okamoto, T. J., Tsuneta, S., Lites, B. W., Kubo, M., Yokoyama, T., Berger, T. E., Ichimoto, K., Katsukawa, Y., Nagata, S. Shibata, K., Shimizu, T., Shine, R. A., Suematsu, Y., Tarbell, T. D., \& Title, A. M. 2009, ApJ, 697, 913

Parker, E. N. 1953, ApJ, 117, 431

Pikel'Ner, S. B. 1971, Sol. Phys., 17, 44

Priest, E. R. 1982, Solar magneto-hydrodynamics (Dordrecht, Holland; Boston: D. Reidel Pub. Co.; Hingham,)

Priest, E. R. \& Smith, E. A. 1979, Sol. Phys., 64, 267

Saito, K. \& Tandberg-Hanssen, E. 1973, Sol. Phys., 31, 105

Schrijver, C. J. 2001, Sol. Phys., 198, 325

Smith, E. A. \& Priest, E. R. 1977, Sol. Phys., 53, 25

Stenborg, G., Vourlidas, A., \& Howard, R. A. 2008, ApJ, 674, 1201

Tandberg-Hanssen, E. 1995, The nature of solar prominences (Dordrecht; Boston: Kluwer)

Titov, V. S., Priest, E. R., \& Demoulin, P. 1993, A\&A, 276, 564

van Ballegooijen, A. A. \& Cranmer, S. R. 2010, ApJ, 711, 164

Wang, J., Zhang, Y., Zhou, G., Harra, L. K., Williams, D. R., \& Jiang, Y. 2007, Sol. Phys., 244, 75

Wang, Y.-M. 1999, ApJ, 520, L71

Wu, S. T., Bao, J. J., An, C. H., \& Tandberg-Hanssen, E. 1990, Sol. Phys., 125, 277

Xia, C., Chen, P. F., Keppens, R., \& van Marle, A. J. 2011, ApJ, 737,27

Zhang, M. \& Low, B. C. 2005, ARA\&A, 43, 103 